\title{
Study on metal corrosion protection mechanism of graphene nano coating
}

\author{
Xuelian Bai ${ }^{1, *}$, Bin Wu ${ }^{1}$, Kun Jiang ${ }^{1}$, Feng Zhang ${ }^{1}$, Hua Zhang ${ }^{1}$, Chengjun Liu ${ }^{1}$, Meijing Cai ${ }^{1}$ \\ ${ }^{1}$ PLA Army Academy of Artillery and Air Defense, Hefei 230031, China
}

\begin{abstract}
Organic coating is considered to be one of the most effective methods to prevent metal corrosion. The corrosion resistance of the coating was tested by the improved copper accelerated acetic acid immersion test, and the quality change of the coating sample before and after immersion was tested. The appearance change of graphene was observed by SEM, and the anticorrosion mechanism of graphene was studied. The results showed that the copper salt accelerated acetic acid salt immersion test accelerated the corrosion process and shortened the determination time. There was no obvious change in the test sample after soaking for $400 \mathrm{~h}$. The main anticorrosion mechanism is that the conductive graphene in graphene nano anticorrosive coating can effectively protect the metal substrate by "conductive bridging".
\end{abstract}

\section{Introduction}

Currently, corrosion costs are increasing, and preventive strategies have become an important industrial demand. Anticorrosive coatings on metal surface can play the role of shielding, passivation and electrochemical protection, which is of great significance to the research of metal corrosion and protection.

Graphene is the thinnest two-dimensional material in the world. It is found that graphene has many advantages in mechanics, electricity, heat and magnetism Their unique nanostructures make them expected to be widely used in high-performance electronic devices, composites, biomaterials and gas sensors ${ }^{[1-2]}$. At the same time, it has become a new favorite in the field of metal corrosion protection because of its good conductivity and physical barrier effect determined by $\mathrm{sp} 2$ hybrid structure. In addition, it has high strength and good tribological properties[3]. At present, a lot of researches were carried out by modifying graphene oxide surface with molecules (silane coupling agent, polymer monomer, etc.) and covering nano particles (nano-SiO2, $\mathrm{TiO} 2$ ) on the surface of graphene oxide ${ }^{[4-5]}$. In this paper, based on the common epoxy zinc rich anticorrosive coatings, by adding non oxidized graphene and silane coupling agent in the preparation process, the compatibility between graphene and epoxy resin is enhanced, and the economic and environmental protection graphene anticorrosive coating is developed ${ }^{[6-7]}$. There are still many problems in the application of graphene in anti-corrosion coatings, especially the anti-corrosion mechanism needs to be further explored. Therefore, a kind of graphene anticorrosion coating is developed in this paper. Aiming at the harsh corrosion environment of amphibious equipment, the copper salt accelerated acetic acid salt soaking method is adopted, and the common metal shell material steel of amphibious equipment is selected to explore the corrosion mechanism.

\section{Experiment}

\subsection{Materials}

Graphene, E-12 epoxy resin, silane coupling agent KH550, 1000 mesh zinc powder, calcium carbonate, mica powder, n-butanol, xylene, propylene glycol methyl ether acetate, isophorone, cyclohexanone, Nmethylpyrrolidone, are all chemically pure. A subsubsection. The paragraph text follows on from the subsubsection heading but should not be in italic.

\subsection{Experiment}

The test solution was prepared according to the method of copper accelerated acetic acid salt spray test (CASS) in GB / $\mathrm{T}$ 10125-976 : dissolve sodium chloride in distilled water to obtain a colorless solution with a concentration of $50 \pm 5 \mathrm{~g} / \mathrm{L}$ and $\mathrm{pH}$ of the solution in the range of 6.0-7.0; and The concentration of copper dichloride is $0.26 \pm 0.02 \mathrm{~g} / \mathrm{l}$ in salt solution, and glacial acetic acid is added to salt solution to ensure $\mathrm{pH}$ value of the prepared solution is 3.0.

The preparation method of graphene nano anticorrosion coating in this study, refer to the patent [8]: 17 parts of epoxy resin and 20 parts of mixed solvent (xylene / n-butanol / propylene glycol methyl ether acetate, volume ratio: 5/3/1) are evenly mixed with highspeed mixer and then added into the paint mixing tank Start the high-speed mixer, add 1 part of dispersant (silane coupling agent KH550), 1 part of leveling agent isophorone, 8 parts of iron red and 11 parts of mica powder. After mixing evenly, grind the fineness of the paint paste to less than $30 \mu \mathrm{m}$, then place it in the mixing

*xlbai@ustc.edu 
tank, turn on the high-speed mixer, add 3 parts of pre dispersed graphene slurry and 15 parts of zinc powder, stir at high speed $(3000 \mathrm{r} / \mathrm{min})$ for $15 \mathrm{~min}$, make up the solvent, adjust to the original solid content, and filter with 100 mesh filter screen to prepare graphene epoxy zinc rich. According to the mass ratio of graphene epoxy zinc rich primer and diluent $70 / 21$, and the mass ratio of graphene epoxy zinc rich primer and curing agent is $70 / 5$, the coating is prepared.

Tinplate, one of the standard test plates specified in GB/T9271-2008 (standard test plates for paints and varnishes), is selected as the test sample material. The prepared coating is applied to the surface of the purchased test grade tinplate, dried at $100{ }^{\circ} \mathrm{C}$ and placed at room temperature for $72 \mathrm{~h}$. The test sample is made, and the coating test plate is weighed, analyzed by SEM, and then fully immersed in the test After the test, take out the test sample and let it stand for $0.5-1 \mathrm{~h}$. then clean or soak the sample in clean flowing water with temperature not exceeding $40{ }^{\circ} \mathrm{C}$, and then blow dry and weigh the sample.

\section{Results and discussion}

\subsection{Anticorrosion performance test of coatings}

Graphene, E-12 epoxy resin, silane coupling agent KH550, 1000 mesh zinc powder, calcium carbonate, mica powder, n-butanol, xylene, propylene glycol methyl ether acetate, isophorone, cyclohexanone, Nmethylpyrrolidone, are all chemically pure. A subsubsection. The paragraph text follows on from the

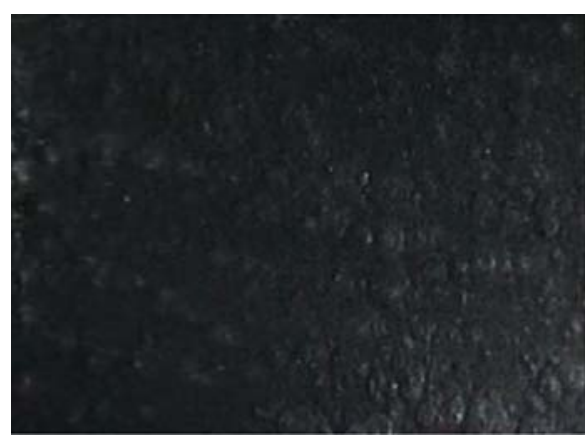

(a) $\mathrm{G}(0 \mathrm{~h})$

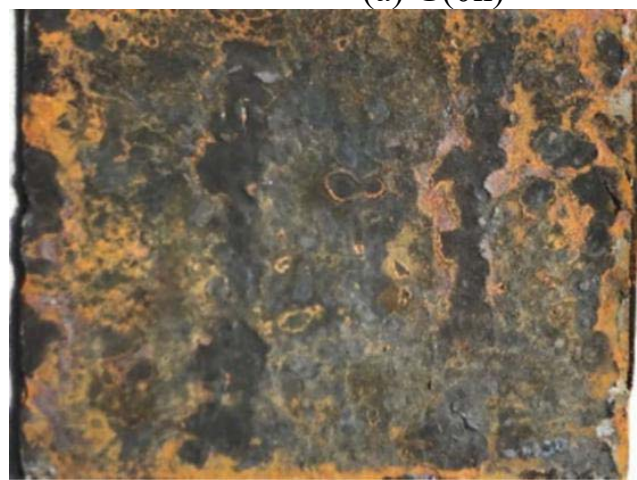

(c) $\mathrm{G}(125 \mathrm{~h})$

Figure1 The appearance of coating sample before and after soaking

Fig. 1 shows the digital photos of the un-soaked $\mathrm{G}(0 \mathrm{~h})$ graphene coating (Fig. 1 (a) and the graphene nano anticorrosion coating G-Zn (0h) (Fig. 1 (b)), as well as the subsubsection heading but should not be in italicAccording to the test method in section 1.2, copper accelerated acetic acid salt solution is prepared, which has stronger corrosion ability than neutral salt water, and the corrosion time is shorter; several test samples are prepared, and their mass $\mathrm{mt} 0$ is weighed respectively, and then the immersion test of $t$ time is conducted, and the immersion time $t$ is $0 \sim 400 \mathrm{~h}$. After the test, take out the test sample, clean and dry according to the test method in section 1.2, and then weigh its mass MT; if the test sample plate is subjected to $25 \mathrm{~h}$ immersion test, the mass of the sample plate made before immersion is M250. The mass of the sample plate after cleaning and drying is $\mathrm{m} 25$, and so on, the quality of the sample plate before and after soaking changes to $\Delta \mathrm{m}(\Delta \mathrm{M}=\mathrm{mt}-\mathrm{mt} 0)$. After soaking, the mass of sample plate increases greatly, indicating the occurrence of corrosion. The $\Delta \mathrm{m}$ of commercially available graphene coatings did not change significantly within $100 \mathrm{~h}$, but increased sharply after $125 \mathrm{~h}$, indicating that serious corrosion had occurred. The self-made graphene nano anti-corrosion coating had no obvious change within $400 \mathrm{~h}$ (Tab.1), which indicated that the corrosion resistance of the coating was long.

Tabel 1 The change of coating sample quality with soaking time before and after soaking

\begin{tabular}{|c|c|c|c|c|c|c|c|c|c|c|c|}
\hline \multicolumn{2}{|c|}{ Time/h } & 0 & \multirow{2}{*}{$\frac{25}{0.02}$} & 50 & 75 & 100 & 125 & 150 & 200 & 300 & 400 \\
\hline \multirow[t]{2}{*}{$\Delta \mathrm{m} / \mathrm{g}$} & $\bar{G}$ & 0 & & 0.00 & $\overline{0} \overline{0} .02$ & $\overline{0} .01$ & 0.08 & - & - & - & - \\
\hline & $\begin{array}{l}\text { G- } \\
\text { Zn }\end{array}$ & 0 & 0.01 & $\overline{0} .01$ & 0.01 & 0.01 & 0.02 & $\overline{0} .02$ & $\overline{0} .02$ & $\overline{0} .02$ & $\overline{0} .03$ \\
\hline
\end{tabular}

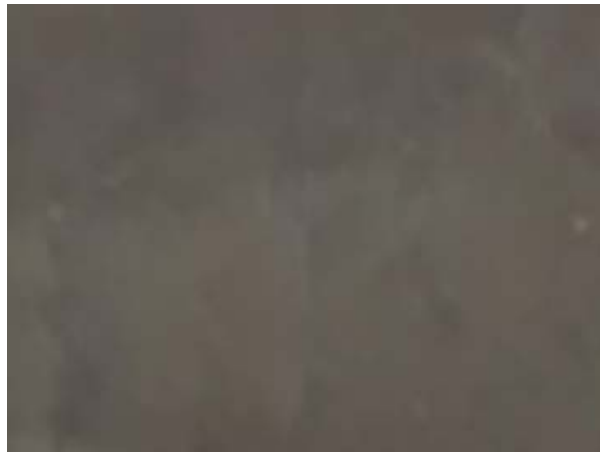

(d) G-Zn(0h)

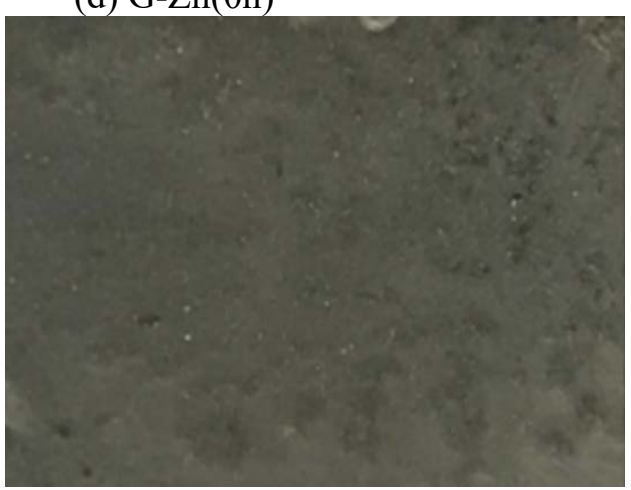

(d) G-Zn(400h)

graphene coating G(Fig. 1(c)) after soaking for $125 \mathrm{~h}$ and the self-made graphene nano anti-corrosion coating after 400h.Digital photo of G-Zn test sample plate (Fig. 1 (d)). 
Comparing the appearance of graphene coating g sample before and after immersion for $125 \mathrm{~h}$ as shown in Fig.1(a) and Fig. 1(c), it is found that there are large areas of blistering, rust, discoloration and other corrosion phenomena after soaking for $125 \mathrm{~h}$. Compare the graphene nano anticorrosive coating samples before and after $400 \mathrm{~h}$ immersion as shown in Fig.1(b) and Fig. 1(d) No obvious pitting, blistering, rust, discoloration and other corrosion phenomena are found on the sample appearance. Therefore, compared with graphene coating $\mathrm{g}$, the coating is more resistant to copper accelerated acetic acid salt corrosion than graphene coating g. the time of corrosion phenomenon of coating test sample is an important technical index to determine the anticorrosion performance of coatings.

The traditional neutral salt spray method (GB/T17712007 determination of resistance to neutral salt spray of paints and varnishes) uses a distilled water solution of

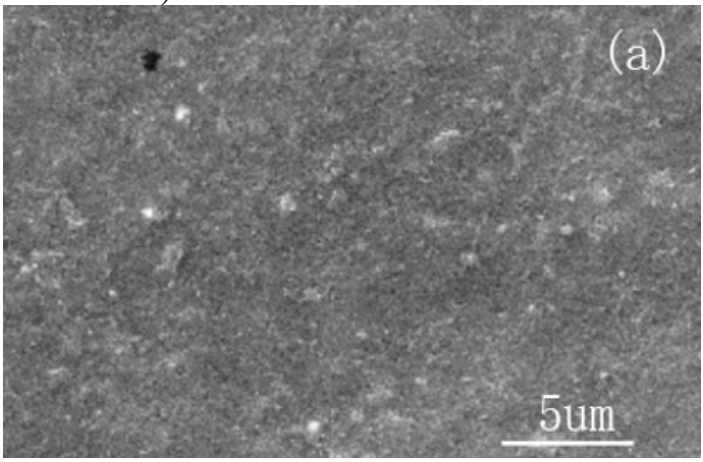

Figure 2 SEM of the test sample(G-Zn) after soaking for $400 \mathrm{~h}$

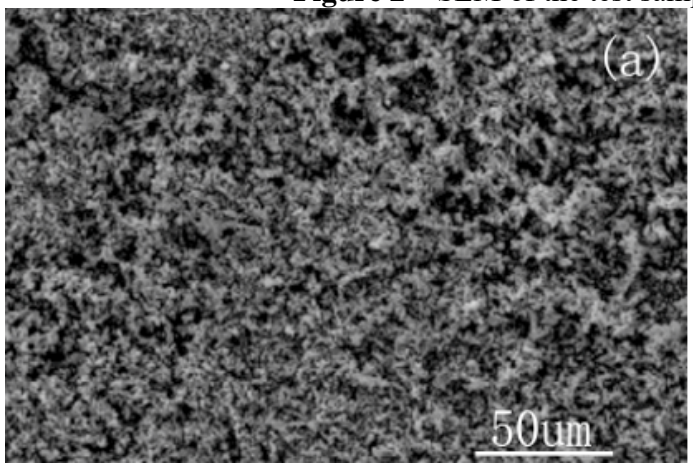

Figure 3 SEM of G sample board after soaking for $125 \mathrm{~h}$

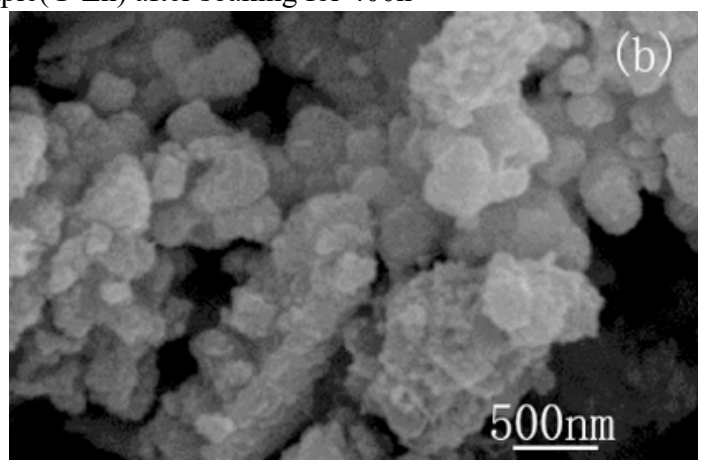

\subsection{Morphology of coating}

The SEM image of the sample after soaking for $400 \mathrm{~h}$ is shown in Fig. 2. The G-Zn sample soaked for $400 \mathrm{~h}$ is intact, and the new nano graphene coating has good anticorrosion effect. The SEM images of commercially available graphene coating $\mathrm{G}$ after soaking for $125 \mathrm{~h}$ are shown in Fig. 3. The surface of the coating is accelerated by the presence of a large amount of salt and salt.

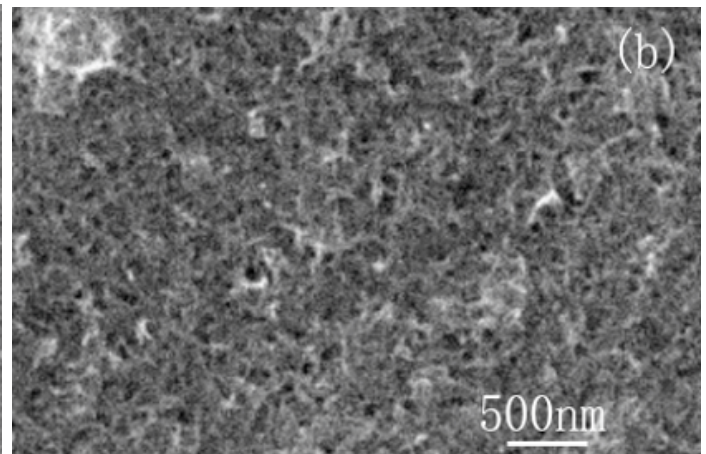

powder, but also ensure the cathodic protection of the coating.

It is considered that graphene can be connected with discontinuous zinc powder to form a conductive network with zinc powder as anode sacrifice, so as to protect the steel substrate as cathode. When some zinc powder in the graphene epoxy zinc rich coating is oxidized to zinc salt and the conductivity decreases, it can also pass through the adjacent layers and the upper layer of $\mathrm{Zn}$ Contact makes the electron transport path bypass the zinc salt and continues to play the role of cathodic protection

\section{Conclusion}

Graphene nano anticorrosive coating was prepared by replacing part of zinc powder with graphene. The anticorrosion performance of the coating was tested by improved copper accelerated acetic acid immersion test 
The weight analysis and SEM analysis of the corrosion surface show that the test sample has no obvious change after soaking for $400 \mathrm{~h}$, which indicates that the copper salt accelerated acetic acid salt immersion test accelerates the corrosion process and shortens the determination time, which is a new and fast test method. The analysis also shows that the main anti-corrosion mechanism of the copper salt accelerated acetate immersion method is graphene nano anti-corrosion. The conductive graphene in the anticorrosive coating can effectively "bridge" the metal matrix, improve the utilization rate of zinc powder and save resources.

\section{References}

1. Nanda S. S., Papaefthymiou G. C. and Dong K. Y. 2015 Functionalization of graphene oxide and its biomedical applications, Rev. Solid State Sci. 40.291-315

2. Jiang F. W., Zhao W. J., Wu Y. M., Dong J. D., Zhou K. H., Lu G. M. and Pu J. B., 2019 Anticorrosion behaviors of epoxy composite coatings enhanced via graphene oxide with different aspect ratios, Prog. Org. Coat. 127.70-9

3. Ramezanzadeh B., Haeri Z. and Ramezanzadeh M., 2016 A facile route of making silica nanoparticlescovered graphene oxide nanohybrids $\left(\mathrm{SiO}_{2}-\mathrm{GO}\right)$; fabrication of $\mathrm{SiO}_{2}-\mathrm{GO} /$ epoxy composite coating with superior barrier and corrosion protection performance, Chem. Eng. J. 303.511-28

4. Haeri S Z, Ramezanzadeh B and Asghari M 2017 A novel fabrication of a high performance $\mathrm{SiO}_{2}$ graphene oxide nanohybrids: Characterization of thermal properties of epoxy nanocomposites filled with $\mathrm{SiO}_{2}-\mathrm{GO}$ nanohybrids J. Colloid Interf. Sci. $493111-22$

5. Krishamoorthy K, Jeyasubramanian K, Preamanathan M, Subbuah G, Shin S H and Kim S J ,2014 Graphene oxide nanopaint, Carbon 72.32837

6. Ramezanzadeh B, Niroumandrad S, Ahmadi A, et al 2016 Enhancement of barrier and corrosion protection performance of an epoxy coating through wet transfer of amino functionalized graphene oxide, Corros. Sci. 103.283-304

7. Hossein H, Mohsen M, Ehsan S and Amin R B 2017 Microstructure, deposition mechanism and corrosion behavior of nanostructured cerium oxide conversion coating modified with chitosan on AA2024 aluminum alloy, J. Alloy. Compd. 725.968-75

8. Jiangsu Dawson New Material Co., Ltd. Zine heavy anticorrosive coating and its preparation method[P].China, Patent of Invention, 201410097011.6,2014-07-02.(Ch) 